Sharif University of Technology
Scientia Iranica
SCIENTIA

\title{
Softening effect of nonlocality against the hardening effect of stretching in a capacitive micro-beam
}

\author{
Sh. Valilou, R. Shabani* and Gh. Rezazadeh \\ Department of Mechanical Engineering, Urmia University, Urmia, Iran.
}

Received 7 October 2014; received in revised form 20 February 2015; accepted 25 April 2015

\section{KEYWORDS}

Nonlocal theory;

Nonlinear dynamics;

Frequency response;

Perturbation method.

\begin{abstract}
This paper investigates the nonlinear resonant behavior of a capacitive microbeam based on the nonlocal theory of elasticity. The micro-beam is deflected by a DC voltage, where it acts as a micro-resonator by superimposing an AC voltage. Taking into account stretching effects, the Galerkin projection method is used to discretize the partial differential equations into a set of nonlinear, ordinary differential equations. The multiple-scales method is used to obtain an approximate analytical solution to construct the nonlinear resonant curves of the transverse vibration amplitude. Taking into account the classical and nonlocal elasticity theories, the frequency response curves are plotted for different values of DC voltage. Effects of mid-plane stretching on the resonant curves are also examined. The hardening behavior of the system is shown to decrease due to the presence of the nonlocality, as well as the DC voltage. However, mid-plane stretching increases the hardening effects. The results show that, despite the existence of nonlinearity in the system, this conflict effect can result in a linear frequency response curve for some values of the nonlocal parameter.
\end{abstract}

(C) 2016 Sharif University of Technology. All rights reserved.

\section{Introduction}

Micro-beams are key components in a large number of micro-electromechanical systems (MEMS) [1]. Microswitches, accelerometers, mass flow sensors, temperature sensors, and micro-resonators are just a few examples of MEMS applications. Electrostatic actuation has been used as a common mode of actuation in MEMS due to its compatibility with micro-fabrication processes [2]. In an electrostatic micro-beam-based resonator, the micro-beam is deflected by a DC bias voltage and then driven to vibrate around its deflected position by an AC harmonic load. There are limits for the applied DC and AC voltages. Thereafter, a pull-in instability takes place [3], which leads to a collapse of the micro-beam and, hence, failure of the device. So, in the design of such devices, it is

*. Corresponding author. Mobile: 09143410618

E-mail address: r.shabani@urmia.ac.ir (R. Shabani) important to tune the electrostatic load so that the micro-beam operates in a safe voltage range, in order to prevent static and dynamic instability. Various papers have been published on the nonlinear dynamics and stability of micro-resonators. Mojahedi et al. [4] investigated the static stability of an electrostatically actuated micro-beam based on the homotopy perturbation method. This method was also employed to pullin the instability of electrostatically actuated carbon nanotubes [5]. In addition, Nayfeh et al. [6] studied the nonlinear frequency response of a micro-resonator and showed that the amplitude of the AC component can change the frequency response effectively. Younis and Nayfeh [7] investigated the effects of axial force and mid-plane stretching on the resonant curves. They showed that DC load can affect the responses, qualitatively and quantitatively, resulting in either a softening or hardening behavior. They also studied the frequency response of micro-resonators due to sub- and superharmonic excitations [8]. 
In an experimental study, Jin and Wang [9] fabricated an electrostatic resonator in single-crystal silicon and explored its second superharmonic resonant behavior. Mestorn et al. [10] reported on both the experimental and theoretical modeling used to study the dynamic behavior of MEMS resonators. The nonlinear response of a resonant micro-beam with purely parametric electrostatic actuation was studied by Rhoads et al. [11]. They modeled the electrostatic interaction between the beam and the multiple electrodes as a parallel-plate capacitor with a single moving plate and minimal fringe field effects. Their results revealed that an inaccurate representation at the modeling stage can lead to an inaccurate prediction of frequency response. Researchers showed experimentally that when the thickness of a beam is in the order of microns and submicrons, it displays a sizedependent deformation behavior $[12,13]$. Consequently, new higher order theories, such as strain gradient, modified couple stress, and micropolar theories, should be employed. Many studies have been undertaken on the size-dependent behavior of micro-beams.

For instance, based on the Euler-Bernoulli theory, Kong et al. [14] investigated the vibrational behavior of micro-beams and reported the size-dependent natural frequencies. Employing both the strain gradient and modified couple stress theories, Akgöz and Civalek $[15,16]$ examined the buckling and free vibrations of a micro-beam. Asghari et al. [17] explored the size-dependent static and dynamic behavior of functionally graded micro-beams on the basis of the modified couple stress theory. Taking into account the nonlinear mid-plane stretching effects, Ghayesh et al. $[18,19]$ investigated the nonlinear forced vibrations of a micro-beam using both the strain gradient and modified couple stress theories and constructed the frequency-response curves of the system. Employing the modified couple stress theory, Ma et al. [20] studied the size-dependent natural frequencies of a Timoshenko micro-beam. Wang et al. [21] examined the size-dependent behavior of a Timoshenko microbeam based on strain gradient elasticity theories. Ansari et al. [22,23] employed the strain gradient theory to study the vibration properties and thermal post-buckling of a functionally-graded Timoshenko micro-beam. Civalek et al. [24,25] used Eringen's non-local elasticity theory to study the vibrational properties and bending analysis of micro-cantilever microtubules. The investigations were continued by Ghayesh et al. [26] who examined the size-dependent frequency response of a nonlinear micro-beam. They used a pseudo-arc length continuation technique to study the frequency response. Asghari et al. [27] and Ramezani [28] developed a nonlinear model of a micro-beam based on the Timoshenko beam theory. They employed the multiple-time scale per- turbation method to study size-dependent dynamic behavior.

Based on the literature review, double-clamped, micro-beam-based resonators suffer from geometric nonlinearity induced by mid-plane stretching. For large deflections, this nonlinearity becomes more significant. In this paper, the conflict effect of mid-plane stretching and nonlocal beam theory on the nonlinear dynamic behavior of a micro-beam is investigated. Taking into account stretching effects and nonlocal elasticity theory [29], the governing equation for the transverse vibrations of an electrostatic micro-resonator is derived. Employing the Galerkin projection method, the nonlinear partial differential equation is discretized into a set of nonlinear, ordinary differential equations. Then the multiple-scales method is used to obtain an approximate analytical solution for the nonlinear resonant curves. Employing classical and nonlocal elasticity theories, the frequency response curves are plotted for different values of DC voltage. The effect of mid-plane stretching on the resonant curves is also examined. Finally, the conflict effect of nonlocality and mid-plane stretching on the frequency response is studied.

\section{Mathematical modeling}

Figure 1 shows a schematic view of an electrostaticallyactuated micro-resonator. The system consists of an elastic beam with length $L$, width $b$, and thickness $h$, with fixed-fixed boundary conditions, which is suspended over a stationary conductor plate. When a voltage is applied between two electrodes, an attractive electrostatic force pulls down the upper deformable electrode.

Taking into account the Euler-Bernoulli beam theory, the displacement field for planar motion of the microbeam can be written as:

$$
u=u_{0}-z \frac{\partial w}{\partial x}, \quad w=w(x, t)
$$

where $u$ and $w$ are the $x$ and $z$ components of the displacement vector, respectively, and $u_{0}$ is the axial displacement of a point on the mid-plane. Based on the nonlocal theory of elasticity, the constitutive relations

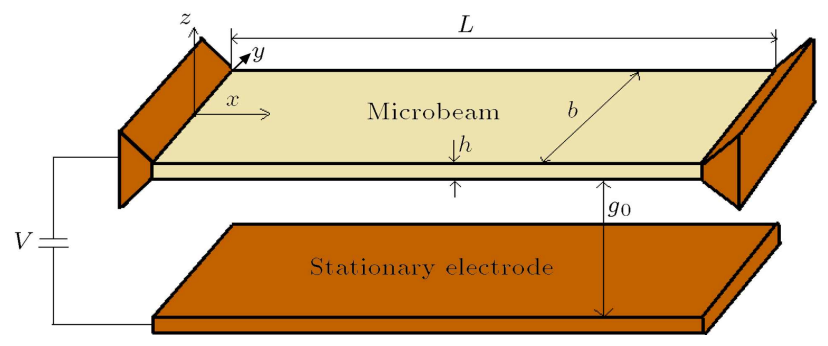

Figure 1. Schematic figure of an electrostatically actuated fixed-fixed micro-beam. 
of the microbeam can be expressed as $[29,30]$ :

$$
\begin{aligned}
& \sigma_{x x}-\mu \frac{\partial^{2} \sigma_{x x}}{\partial x^{2}}=E \varepsilon_{x x}, \\
& \varepsilon_{x x}=-z \frac{\partial^{2} x}{\partial x^{2}} \Rightarrow \\
& \sigma_{x x}-\mu \frac{\partial^{2} \sigma_{x x}}{\partial x^{2}}=-E z \frac{\partial^{2} w}{\partial x^{2}},
\end{aligned}
$$

where $\varepsilon$ and $\sigma$ are the strain and stress tensors, respectively. $E$ is Young's modulus and $\mu=\left(e_{0} l\right)^{2}$ is the nonlocal parameter. $l$ is the length scale parameter and $e_{0}$ is a material constant. Considering mid-plane stretching effects, the nonlocal theory of elasticity, and, employing Hamilton's variational principle, the governing equation for transverse vibrations of the micro-beam can be written as $[29,30]$ :

$$
\begin{aligned}
E I & \frac{\partial^{4} w(x, t)}{\partial x^{4}}+\rho A \frac{\partial^{2} w(x, t)}{\partial t^{2}}+\mu\left(\frac{-3 \varepsilon b V^{2}}{\left(g_{0}+w\right)^{4}}\left(\frac{\partial w}{\partial x}\right)^{2}\right. \\
& \left.+\frac{\varepsilon b V^{2}}{\left(g_{0}+w\right)^{3}} \frac{\partial^{2} w}{\partial x^{2}}-\rho A \frac{\partial^{4} w}{\partial x^{2} \partial t^{2}}\right) \\
& -\left(\frac{E A}{2 l} \int_{0}^{l}\left(\frac{\partial w(x, t)}{\partial x}\right)^{2} d x\right) \frac{\partial^{2} w}{\partial x^{2}}+c \frac{\partial w}{\partial t} \\
& =\frac{\varepsilon_{0} b V^{2}}{2\left(g_{0}+w\right)^{2}}
\end{aligned}
$$

where $E I, \rho$, and $A$ are the flexural rigidity, density, and cross section of the micro-beam, respectively. The right hand term in Eq. (3) reveals the nonlinear attractive electrostatic force and $g_{0}$ denotes the initial gap between the fixed and moving electrodes. In order to get the nondimensional equation of motion, the following dimensionless parameters are defined as:

$$
\begin{aligned}
& \hat{w}=\frac{w}{g_{0}}, \quad \hat{x}=\frac{x}{L}, \\
& \hat{t}=\frac{t}{\sqrt{\rho A L^{4} / E I}}, \quad \hat{\omega}=\frac{\omega}{\sqrt{E I / \rho A L^{4}}},
\end{aligned}
$$

where $\omega$ is the fundamental frequency of the system. Substituting these parameters into Eq. (3) and ignoring the hat notation for briefness results in the following dimensionless nonlinear governing equation:

$$
\begin{aligned}
\frac{\partial^{4} w(x, t)}{\partial x^{4}} & +\frac{\partial^{2} w(x, t)}{\partial t^{2}}-D_{1} \Gamma(w, w) \frac{\partial^{2} w(x, t)}{\partial x^{2}}+c \frac{\partial w}{\partial t} \\
& +D_{2} \frac{V^{2}}{(1+w)^{4}}\left(\frac{\partial w}{\partial x}\right)^{2}+D_{3} \frac{V^{2}}{(1+w)^{3}} \frac{\partial^{2} w}{\partial x^{2}} \\
& +D_{4} \frac{\partial^{4} w}{\partial x^{2} \partial t^{2}}=D_{5} \frac{V^{2}}{(1+w)^{2}}
\end{aligned}
$$

where:

$$
\begin{aligned}
& D_{1}=\frac{6 g_{0}^{2}}{h^{2}}, \\
& D_{2}=\frac{-3 \mu \varepsilon b L^{2}}{E I g_{0}^{3}}, \\
& D_{3}=\frac{\mu \varepsilon b L^{2}}{E I g_{0}^{3}}, \\
& D_{4}=\frac{\mu}{L^{2}}, \\
& D_{5}=\frac{\varepsilon b L^{4}}{2 E I g_{0}^{3}}, \\
& \Gamma\left(f_{1}, f_{2}\right)=\int_{0}^{l} \frac{\partial f_{1}}{\partial x} \frac{\partial f_{2}}{\partial x} d x .
\end{aligned}
$$

In the numerical simulations, it is considered that the micro-beam is deflected by a DC voltage, $V_{d c}$. Then, the forced response of the system is considered regarding these conditions by superimposing a harmonic AC voltage with amplitude $V_{a c}$. So, the total deflection of the micro-beam consists of two parts, as:

$$
w(x, t)=w_{s}(x)+w_{d}(x, t),
$$

in which $w_{s}(x)$ introduces the static deflection of the micro-beam and $w_{d}(x, t)$ denotes the dynamic deflection about $w_{s}(x)$. The governing equation for the static deflection can be obtained by dropping the time in Eq. (5) as:

$$
\begin{gathered}
\frac{\partial^{4} w_{s}}{\partial x^{4}}-D_{1} \Gamma\left(w_{s}, w_{s}\right) \frac{\partial^{2} w_{s}}{\partial x^{2}}+D_{2} \frac{V_{d c}^{2}}{\left(1+w_{s}\right)^{4}}\left(\frac{\partial w_{s}}{\partial x}\right)^{2} \\
+D_{3} \frac{V_{d c}^{2}}{\left(1+w_{s}\right)^{3}} \frac{\partial^{2} w_{s}}{\partial x^{2}}=D_{5} \frac{V_{d c}^{2}}{\left(1+w_{s}\right)^{2}}
\end{gathered}
$$

This equation has four nonlinear terms in which the Taylor expansion can be applied to treat these terms, as follows:

$$
\begin{aligned}
F(V, w)= & F\left(V_{d c}, w_{s}\right)+\frac{\partial F}{\partial V_{d c}} \delta V+\frac{1}{2} \frac{\partial^{2} F}{\partial V_{d c}^{2}} \delta V^{2} \\
& +\frac{\partial F}{\partial w_{s}} \delta w+\frac{1}{2} \frac{\partial^{2} F}{\partial w_{s}^{2}} \delta w^{2}+\frac{1}{6} \frac{\partial^{3} F}{\partial w_{s}^{3}} \delta w^{3} \\
& +\frac{\partial^{2} F}{\partial V_{d c} \partial w_{s}} \delta V \delta w+\frac{1}{2} \frac{\partial^{3} F}{\partial V_{d c}^{2} \partial w_{s}} \delta V^{2} \delta w \\
& +\frac{1}{2} \frac{\partial^{3} F}{\partial V_{d c} \partial w_{s}^{2}} \delta V \delta w^{2} .
\end{aligned}
$$

Considering this expansion, the governing equation can 
be written as:

$$
\begin{aligned}
& \frac{\partial^{4} w_{d}}{\partial x^{4}}+\frac{\partial^{2} w_{d}}{\partial t^{2}}-D_{1}\left\{\left[\Gamma\left(w_{s}, w_{s}\right)+2 \Gamma\left(w_{s}, w_{d}\right)\right] \frac{\partial^{2} w_{d}}{\partial x^{2}}\right. \\
& +\left[2 \Gamma\left(w_{s}, w_{d}\right)+\Gamma\left(w_{d}, w_{d}\right)\right] \frac{\partial^{2} w_{s}}{\partial x^{2}} \\
& \left.+\Gamma\left(w_{d}, w_{d}\right) \frac{\partial^{2} w_{d}}{\partial x^{2}}\right\}+c \frac{\partial w_{d}}{\partial t} \\
& +D_{2}\left\{\frac{-4 V_{d c}^{2}}{\left(1+w_{s}\right)^{5}}\left(\frac{\partial w_{s}}{\partial x}\right)^{2} w_{d}\right. \\
& +\frac{10 V_{d c}^{2}}{\left(1+w_{s}\right)^{6}}\left(\frac{\partial w_{s}}{\partial x}\right)^{2} w_{d}^{2}-\frac{20 V_{d c}^{2}}{\left(1+w_{s}\right)^{7}}\left(\frac{\partial w_{s}}{\partial x}\right)^{2} w_{d}^{3} \\
& +\frac{2 V_{d c} V_{a c}}{\left(1+w_{s}\right)^{4}}\left(\frac{\partial w_{s}}{\partial x}\right)^{2}+\frac{V_{a c}^{2}}{\left(1+w_{s}\right)^{4}}\left(\frac{\partial w_{s}}{\partial x}\right)^{2} \\
& -\frac{8 V_{d c} V_{a c}}{\left(1+w_{s}\right)^{5}}\left(\frac{\partial w_{s}}{\partial x}\right)^{2} w_{d}-\frac{4 V_{a c}^{2}}{\left(1+w_{s}\right)^{5}} \\
& \left.\left(\frac{\partial w_{s}}{\partial x}\right)^{2} w_{d}\right\}+D_{3}\left\{\frac{-3 V_{d c}^{2}}{\left(1+w_{s}\right)^{4}} \frac{\partial^{2} w_{s}}{\partial x^{2}} w_{d}\right. \\
& +\frac{6 V_{d c}^{2}}{\left(1+w_{s}\right)^{5}} \frac{\partial^{2} w_{s}}{\partial x^{2}} w_{d}^{2}-\frac{10 V_{d c}^{2}}{\left(1+w_{s}\right)^{6}} \frac{\partial^{2} w_{s}}{\partial x^{2}} w_{d}^{3} \\
& +\frac{2 V_{d c} V_{a c}}{\left(1+w_{s}\right)^{3}} \frac{\partial^{2} w_{s}}{\partial x^{2}}+\frac{V_{a c}^{2}}{\left(1+w_{s}\right)^{3}} \frac{\partial^{2} w_{s}}{\partial x^{2}} \\
& \left.-\frac{6 V_{d c} V_{a c}}{\left(1+w_{s}\right)^{4}} \frac{\partial^{2} w_{s}}{\partial x^{2}} w_{d}-\frac{3 V_{a c}^{2}}{\left(1+w_{s}\right)^{4}} \frac{\partial^{2} w_{s}}{\partial x^{2}} w_{d}\right\} \\
& +D_{4} \frac{\partial^{4} w_{d}}{\partial x^{2} \partial t^{2}}=D_{5}\left\{\frac{-2 V_{d c}^{2}}{\left(1+w_{s}\right)^{3}} w_{d}\right. \\
& +\frac{3 V_{d c}^{2}}{\left(1+w_{s}\right)^{4}} w_{d}^{2}-\frac{4 V_{d c}^{2}}{\left(1+w_{s}\right)^{5}} w_{d}^{3}+\frac{2 V_{d c} V_{a c}}{\left(1+w_{s}\right)^{2}} \\
& \left.-\frac{4 V_{d c} V_{a c}}{\left(1+w_{s}\right)^{3}} w_{d}+\frac{V_{a c}^{2}}{\left(1+w_{s}\right)^{2}}-\frac{2 V_{a c}^{2}}{\left(1+w_{s}\right)^{3}} w_{d}\right\} \text {, }
\end{aligned}
$$

where $V_{a c}$ denotes the harmonic component of the driving voltage, ' $\delta V$ ', and $V_{d c}$ is the applied bias voltage.

\section{Perturbation analysis}

In order to solve the governing equation, Galerkin's projection method is employed to reduce it into a set of nonlinear ordinary differential equations with finite degrees of freedom. So, the dynamic displacement of the system is assumed as the following series expan- sions:

$$
w_{d}(x, t)=\sum_{j=1}^{n} \varphi_{j}(x) q_{j}(t),
$$

where $q_{j}(t)$ denotes the generalized coordinates and $\varphi_{j}(x)$ represents the eigenfunctions for the transverse vibrations of a clamped-clamped micro-beam, which can be written as:

$$
\begin{aligned}
\varphi_{j}(x)= & \left(\cos \lambda_{j} x-\cosh \lambda_{j} x-\frac{\cos \lambda_{j} L-\cosh \lambda_{j} L}{\sin \lambda_{j} L-\sinh \lambda_{j} L}\right. \\
& \left.\times\left(\sin \lambda_{j} x-\sinh \lambda_{j} x\right)\right),
\end{aligned}
$$

in which $\lambda_{j}^{\prime} s$ denote the eigenvalues of the micro-beam. Substituting Eq. (11) into Eq. (10) and making use of the orthogonality of trigonometric functions, the following equation is obtained:

$$
\begin{aligned}
& \sum_{j=1}^{n} M_{i j} \ddot{q}_{j}(t)+\sum_{j=1}^{n} C_{i j} \dot{q}_{j}(t)+\sum_{j=1}^{n} K_{i j} q_{j}(t) \\
& \quad+\sum_{j=1}^{n} \sum_{k=1}^{n} K_{i j k} q_{j}(t) q_{k}(t) \\
& \quad+\sum_{j=1}^{n} \sum_{k=1}^{n} \sum_{m=1}^{n} K_{i j k m} q_{j}(t) q_{k}(t) q_{m}(t) \\
& \quad=F_{i} V_{0} \cos \Omega t+f_{i} V_{0}^{2} \cos ^{2} \Omega t \\
& \quad+V_{0} \cos \Omega t \sum_{j=1}^{n} F_{i j} q_{j}(t)+V_{0}^{2} \cos ^{2} \Omega t \sum_{j=1}^{n} f_{i j} q_{j}(t)
\end{aligned}
$$

where $\Omega$ and $V_{0}$ are the nondimensional excitation frequency and harmonic forcing amplitude, respectively. The coefficients in Eq. (13) can be defined as:

$$
\begin{aligned}
M_{i j}= & \int_{0}^{1} \varphi_{j}(x) \varphi_{i}(x) d x+D_{4} \int_{0}^{1} \varphi_{j}^{\prime \prime}(x) \varphi_{i}(x) d x \\
C_{i j}= & \hat{c} \int_{0}^{1} \varphi_{j}(x) \varphi_{i}(x) d x \\
K_{i j}= & \int_{0}^{1} \varphi_{j}^{i v}(x) \varphi_{i}(x) d x \\
& -D_{1} \Gamma\left(w_{s}, w_{s}\right) \int_{0}^{1} \varphi_{j}^{\prime \prime}(x) \varphi_{i}(x) d x \\
& -2 D_{1} \Gamma\left[w_{s}, \varphi_{j}(x)\right] \int_{0}^{1} \varphi_{i}(x) w_{s}^{\prime \prime} d x \\
& -4 D_{2} V_{d c}^{2} \int_{0}^{1} \frac{\varphi_{j}(x) \varphi_{i}(x)}{\left(1+\hat{w}_{s}\right)^{5}}\left(w_{s}^{\prime}\right)^{2} d x
\end{aligned}
$$




$$
\begin{aligned}
& -3 D_{3} V_{d c}^{2} \int_{0}^{1} \frac{\varphi_{j}(x) \varphi_{i}(x)}{\left(1+\hat{w}_{s}\right)^{4}} w_{s}^{\prime \prime} d x \\
& +2 D_{5} V_{d c}^{2} \int_{0}^{1} \frac{\varphi_{j}(x) \varphi_{i}(x)}{\left(1+\hat{w}_{s}\right)^{3}} d x \\
& K_{i j k}=-2 D_{1} \Gamma\left[w_{s}, \varphi_{k}(x)\right] \int_{0}^{1} \varphi_{j}^{\prime \prime}(x) \varphi_{i}(x) d x \\
& -D_{1} \Gamma\left[\varphi_{j}(x), \varphi_{k}(x)\right] \int_{0}^{1} \varphi_{i}(x) w_{s}^{\prime \prime} d x \\
& +10 D_{2} V_{d c}^{2} \int_{0}^{1} \frac{\varphi_{j}(x) \varphi_{i}(x) \varphi_{k}(x)}{\left(1+w_{s}\right)^{6}}\left(w_{s}^{\prime}\right)^{2} d x \\
& +6 D_{3} V_{d c}^{2} \int_{0}^{1} \frac{\varphi_{j}(x) \varphi_{i}(x) \varphi_{k}(x)}{\left(1+\hat{w}_{s}\right)^{5}} w_{s}^{\prime \prime} d x \\
& -3 D_{5} V_{d c}^{2} \int_{0}^{1} \frac{\varphi_{j}(x) \varphi_{i}(x) \varphi_{k}(x)}{\left(1+w_{s}\right)^{4}} d x \\
& K_{i j k m}=-D_{1} \Gamma\left[\varphi_{k}(x), \varphi_{m}(x)\right] \int_{0}^{1} \varphi_{j}^{\prime \prime}(x) \varphi_{i}(x) d x \\
& -20 D_{2} V_{d c}^{2} \int_{0}^{1} \frac{\varphi_{i}(x) \varphi_{m}(x) \varphi_{k}(x) \varphi_{j}(x)}{\left(1+\hat{w}_{s}\right)^{7}} \\
& \left(w_{s}^{\prime}\right)^{2} d x-10 D_{3} V_{d c}^{2} \int_{0}^{1} \\
& \frac{\varphi_{i}(x) \varphi_{m}(x) \varphi_{k}(x) \varphi_{j}(x)}{\left(1+w_{s}\right)^{6}} w_{s}^{\prime \prime} d \hat{x} \\
& +4 D_{5} V_{d c}^{2} \int_{0}^{1} \frac{\varphi_{i}(x) \varphi_{m}(x) \varphi_{k}(x) \varphi_{j}(x)}{\left(1+w_{s}\right)^{5}} d x \\
& F_{i}=-2 D_{2} V_{d c} \int_{0}^{1} \frac{\varphi_{i}(x)}{\left(1+w_{s}\right)^{4}}\left(w_{s}^{\prime}\right)^{2} d x \\
& -2 D_{3} V_{d c} \int_{0}^{1} \frac{\varphi_{i}(x)}{\left(1+w_{s}\right)^{3}} w_{s}^{\prime \prime} d x \\
& +2 D_{5} V_{d c} \int_{0}^{1} \frac{\varphi_{i}(x)}{\left(1+w_{s}\right)^{2}} d x \\
& f_{i}=-D_{2} \int_{0}^{1} \frac{\varphi_{i}(x)}{\left(1+w_{s}\right)^{4}}\left(w_{s}^{\prime}\right)^{2} d x \\
& -D_{3} \int_{0}^{1} \frac{\varphi_{i}(x)}{\left(1+w_{s}\right)^{3}} w_{s}^{\prime \prime} d x+D_{5} \int_{0}^{1} \frac{\varphi_{i}(x)}{\left(1+w_{s}\right)^{2}} d x \\
& F_{i j}=8 D_{2} V_{d c} \int_{0}^{1} \frac{\varphi_{j}(x) \varphi_{i}(x)}{\left(1+w_{s}\right)^{5}}\left(w_{s}^{\prime}\right)^{2} d x
\end{aligned}
$$

$$
\begin{gathered}
+6 D_{3} V_{d c} \int_{0}^{1} \frac{\varphi_{j}(x) \varphi_{i}(x)}{\left(1+w_{s}\right)^{4}} w_{s}^{\prime \prime} d x \\
-4 D_{5} V_{d c} \int_{0}^{1} \frac{\varphi_{j}(x) \varphi_{i}(x)}{\left(1+w_{s}\right)^{3}} d x \\
f_{i j}=4 D_{2} \int_{0}^{1} \frac{\varphi_{j}(x) \varphi_{i}(x)}{\left(1+w_{s}\right)^{5}}\left(w_{s}^{\prime}\right)^{2} d x \\
+3 D_{3} \int_{0}^{1} \frac{\varphi_{j}(x) \varphi_{i}(x)}{\left(1+w_{s}\right)^{4}} w_{s}^{\prime \prime} d x \\
-2 D_{5} \int_{0}^{1} \frac{\varphi_{j}(x) \varphi_{i}(x)}{\left(1+w_{s}\right)^{3}} d x
\end{gathered}
$$

Symmetric electrostatic loading of the micro-beam reveals that the first mode can be considered the dominant mode [7]. Therefore, considering the first as the dominant mode yields:

$$
\begin{aligned}
M \ddot{q} & +C \dot{q}+K_{1} q+K_{2} q^{2}+K_{3} q^{3}=F_{1} V_{0} \cos \Omega t \\
& +f_{1} V_{0}^{2} \cos ^{2} \Omega t+F_{2} q V_{0} \cos \Omega t+f_{2} q V_{0}^{2} \cos ^{2} \Omega t
\end{aligned}
$$

where:

$$
\begin{aligned}
& M=M_{11}, \quad C=C_{11}, \quad K_{1}=K_{11}, \quad K_{2}=K_{111}, \\
& K_{3}=K_{1111}, \quad F_{2}=F_{11}, \quad f_{2}=f_{11} .
\end{aligned}
$$

To solve Eq. (15), the method of multiple-scales is employed by assuming a uniform approximate solution in the following form:

$$
\begin{aligned}
q(t ; \varepsilon)= & \varepsilon q_{1}\left(T_{0}, T_{1}, T_{2}\right)+\varepsilon^{2} q_{2}\left(T_{0}, T_{1}, T_{2}\right) \\
& +\varepsilon^{3} q_{3}\left(T_{0}, T_{1}, T_{2}\right)+\ldots,
\end{aligned}
$$

where $T_{0}=t$ is a fast time scale and $T_{1}=\varepsilon t$, and $T_{2}=$ $\varepsilon t$ are slow time scales associated with modulations in the amplitude and phase caused by nonlinearities, damping and any possible resonance. The derivatives, with respect to time, are expressed in terms of the new time scales as:

$$
\begin{aligned}
& \frac{d}{d t}=D_{0}+\varepsilon D_{1}+\varepsilon^{2} D_{2}+\ldots, \\
& \frac{d^{2}}{d t^{2}}=D_{0}^{2}+2 \varepsilon D_{0} D_{1}+\varepsilon^{2} D_{1}^{2}+2 \varepsilon^{2} D_{0} D_{2}+\ldots,
\end{aligned}
$$

where $\varepsilon$ is a small non-dimensional bookkeeping parameter and $D_{n}=\partial / \partial t_{n}$. Taking into account the low damping ratio and forcing amplitude as $C_{i j}=\varepsilon^{2} C_{i j}$, $V_{a c}=\varepsilon^{3} V_{a c}$, substituting Eq. (17) into Eq. (15), and equating coefficients of like powers of $\varepsilon$ on both sides, yield the following set of ODEs:

$$
O(\varepsilon): \quad D_{0}^{2} q_{1}+\omega^{2} q_{1}=0
$$




$$
\begin{aligned}
O\left(\varepsilon^{2}\right): \quad D_{0}^{2} q_{2} & +\omega^{2} q_{2}=-2 D_{0} D_{1} q_{1}-K_{2} q_{1}^{2} \\
O\left(\varepsilon^{3}\right): \quad D_{0}^{2} q_{3} & +\omega^{2} q_{3}=-2 D_{0} D_{1} q_{2}-2 D_{0} D_{2} q_{1} \\
- & D_{1}^{2} q_{1}-C D_{0} q_{1}-2 K_{2} q_{1} q_{2} \\
- & K_{3} q_{1}^{3}+F_{1} V_{0} \cos \left(\Omega T_{0}\right) .
\end{aligned}
$$

The general solution of Eq. (19) can be written as:

$$
q_{1}=A\left(T_{1}, T_{2}\right) e^{i \omega T_{0}}+\bar{A}\left(T_{1}, T_{2}\right) e^{-i \omega T_{0}},
$$

where $A$ is an unknown complex function, and $\bar{A}$ is the complex conjugates of $A$. It can be determined by eliminating the secular and small-divisor terms at the next approximation stage. Substituting Eq. (22) into Eq. (20) leads to the solution of Eq. (21) as:

$$
q_{2}=\frac{K_{2}}{3 \omega^{2}}\left(A^{2} e^{2 i \omega T_{0}}+\bar{A}^{2} e^{-2 i \omega T_{0}}\right)-\frac{2 K_{2}}{\omega^{2}} A \bar{A}
$$

Now, substituting Eq. (23) into Eq. (21) and expressing $\cos \left(\Omega T_{0}\right)$ in complex form yields:

$$
\begin{aligned}
D_{0}^{2} q_{3} & +\omega^{2} q_{3}=-2\left(\frac{d A\left(T_{2}\right)}{d T_{2}} i \omega e^{i \omega T_{0}}\right. \\
& \left.-\frac{\overline{d A}\left(T_{2}\right)}{d T_{2}} i \omega e^{-i \omega T_{0}}\right)-C\left(A\left(T_{2}\right) i \omega e^{i \omega T_{0}}\right. \\
& \left.+\bar{A}\left(T_{2}\right) i \omega e^{-i \omega T_{0}}\right)-2 K_{2}\left(A\left(T_{2}\right) e^{i \omega T_{0}}\right. \\
& \left.+\bar{A}\left(T_{2}\right) e^{-i \omega T_{0}}\right)\left(\frac { K _ { 2 } } { 3 \omega ^ { 2 } } \left(A^{2}\left(T_{2}\right) e^{2 i \omega T_{0}}\right.\right. \\
& \left.\left.+\bar{A}^{2}\left(T_{2}\right) e^{-2 i \omega T_{0}}\right)-\frac{2 K_{2}}{\omega^{2}} A\left(T_{2}\right) \bar{A}\left(T_{2}\right)\right) \\
& -K_{3}\left(A\left(T_{2}\right) e^{i \omega T_{0}}+\bar{A}\left(T_{2}\right) e^{-i \omega T_{0}}\right)^{3} \\
& +\frac{F_{1} V_{0}}{2}\left(e^{i \Omega T_{0}}+e^{-i \Omega T_{0}}\right)
\end{aligned}
$$

In order to describe the closeness of the excitation frequency, $\Omega$, to the fundamental frequency, $\omega$, the detuning parameter, $\sigma$, is defined as:

$$
\Omega=\omega+\varepsilon^{2} \sigma .
$$

Substituting Eqs. (23) and (25) into Eq. (24), the secular terms can be detected by comparing the homogenous solution and the forcing terms. Therefore:

$$
\begin{aligned}
-C i \omega A\left(T_{2}\right) & -2 \frac{\partial A\left(T_{2}\right)}{\partial T_{2}} i \omega+4 \frac{K_{2}^{2}}{\omega^{2}} A^{2}\left(T_{2}\right) \bar{A}\left(T_{2}\right) \\
& -\frac{2 K_{2}^{2}}{3 \omega^{2}} A^{2}\left(T_{2}\right) \bar{A}\left(T_{2}\right)-3 K_{3} A^{2}\left(T_{2}\right) \bar{A}\left(t_{2}\right) \\
& +F_{1} \frac{V_{0}}{2} e^{i \sigma T_{2}}=0 .
\end{aligned}
$$

To find the steady state solution for the transverse vibrations of the micro-beam, the complex function, $A$, is assumed as:

$$
A\left(T_{2}\right)=\frac{1}{2} a e^{i \beta}
$$

where $a$ is the transverse vibration amplitude and $\beta$ is the phase angle, both being real functions of $T_{2}$. Substituting Eq. (27) into Eq. (26) and separating the result into real and imaginary parts, one can obtain:

$$
\left\{\begin{array}{l}
a^{\prime}=\frac{1}{\omega}\left(-C \omega \frac{1}{2} a+F_{1} \frac{V_{0}}{2} \sin (\gamma)\right) \\
\gamma^{\prime}=\frac{1}{\omega a}\left(\omega a \sigma+a^{3}\left(\frac{5}{12} \frac{K_{2}^{2}}{\omega^{2}}-\frac{3}{8} K_{3}\right)+F_{1} \frac{V_{0}}{2} \cos (\gamma)\right),
\end{array}\right.
$$

where autonomous Eqs. (28) is obtained by letting $\gamma=$ $\sigma T_{2}-\beta$. To find the steady state solution, the singular points should be located and the motion in their neighborhoods examined. The stability of the steady state motion shows whether a small perturbation near the points decays or grows. The steady state vibrations occur when $a^{\prime}=\gamma^{\prime}=0$, which corresponds to the singular points of Eq. (28), or to the solutions of:

$$
\left\{\begin{array}{l}
\frac{1}{\omega}\left(-C \omega \frac{1}{2} a+F_{1} \frac{V_{0}}{2} \sin (\gamma)\right)=0 \\
\frac{1}{\omega a}\left(\omega a \sigma+a^{3}\left(\frac{5}{12} \frac{K_{2}^{2}}{\omega^{2}}-\frac{3}{8} K_{3}\right)+F_{1} \frac{V_{0}}{2} \cos (\gamma)\right)=0
\end{array}\right.
$$

Eliminating $\gamma$ in Eq. (28), one can find the frequency response equation as:

$$
4\left(\omega a \sigma+a^{3}\left(\frac{5}{12} \frac{K_{2}^{2}}{\omega^{2}}-\frac{3}{8} K_{3}\right)\right)^{2}+(C \omega a)^{2}=\left(F_{1} V_{0}\right)^{2} .
$$

Eq. (29) reveals that the amplitude of the periodic solution is a function of the detuning parameter, $\sigma$, as a representative of the excitation frequency, the quadratic and cubic stiffness, $K_{2}$ and $K_{3}$, the damping coefficient, $C$, and the amplitude of the harmonic excitation, $V_{0}$. The stability of the steady state solution depends on the eigenvalues of the state equation 'Eq. (28)', which is evaluated at the singular points.

\section{Numerical results and discussion}

In order to validate the correctness of the formulation, the fixed points of the system are compared with those obtained by Nayfeh et al. [8], where the micro-beam specifications were assumed to be $L=510 \mu \mathrm{m}, b=$ $100 \mu \mathrm{m}, g_{0}=1.18 \mu \mathrm{m}, N=8.7$ and $h=1.5 \mu \mathrm{m}$. Figure 2 shows the fixed points of the system versus the applied DC voltage. The stable (upper) and unstable (lower) branches of the equilibrium points collide at a saddle-node bifurcation point (static pull-in instability) at $V_{d c} \approx 4.8 \mathrm{~V}$, which is in close agreement with the result reported in [8]. 


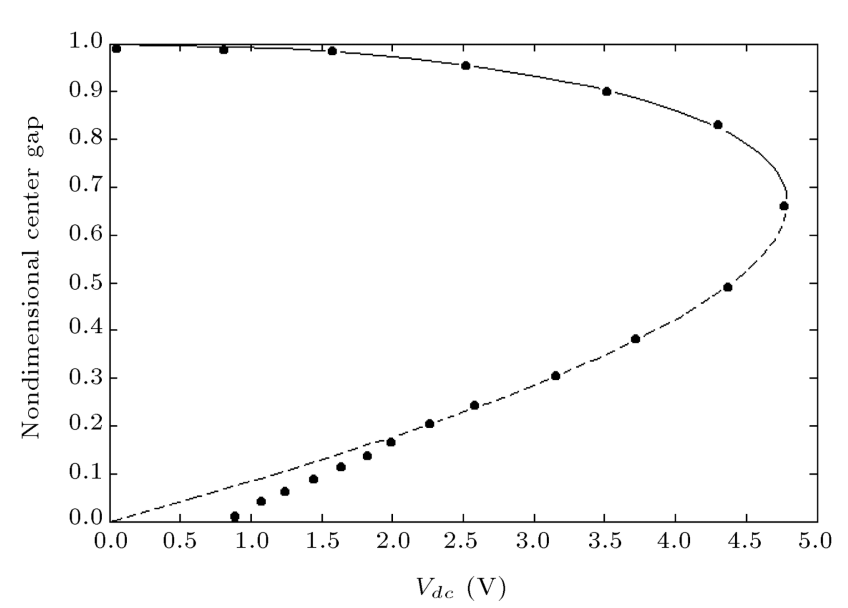

Figure 2. Comparison of the fixed points of the micro-beam versus DC voltage with those obtained by Nayfeh et al. [8] (discrete points).

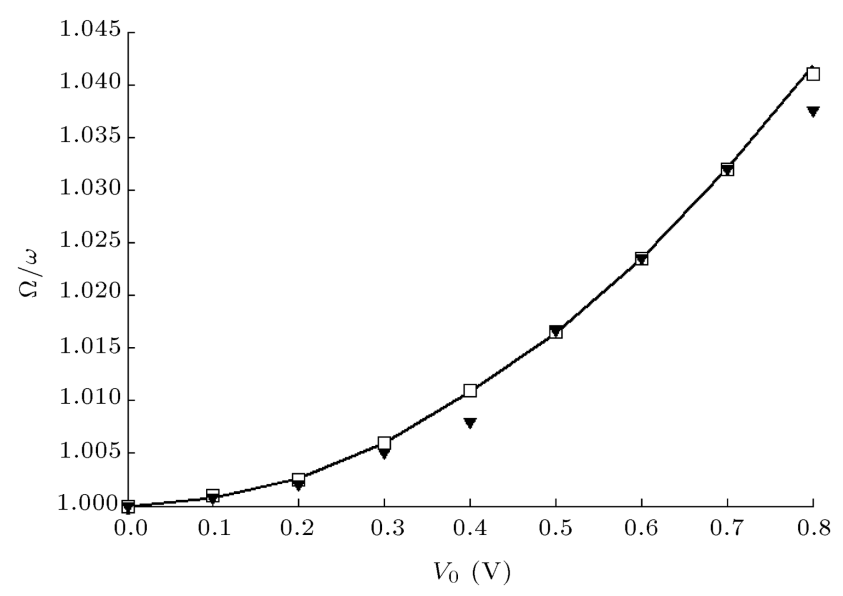

Figure 3. Comparison of the obtained normalized nonlinear resonance frequencies with those obtained theoretically by Nayfeh et al. [7] (squares) and experimentally reported by Tilmans and Lectenberg [31] (triangles).

In addition, the normalized nonlinear resonance frequencies $\left(\Omega_{r} / \mathbf{w}\right)$ are compared with those obtained theoretically by Younis et al. [7] and experimentally reported by Tilmans and Lectenberg [31], where $L=$ $310 \mu \mathrm{m}, Q=197$, and the axial load is set at $0.0009 \mathrm{~N}$, as shown in Figure 3. As can be inferred from the figure, the results are in close agreement.

The material of the micro-beam is epoxy, and its geometrical and material properties used in the simulations are listed in Table 1 . It should be noted that in all simulations, the harmonic forcing amplitude and quality factor are assumed to be $V_{0}=0.1 \mathrm{~V}$ and $Q=100$, respectively. Taking into account the classical theory and imposing different DC voltages, variations in the steady-state amplitude versus the detuning parameter are presented in Figure 4.

The figure shows that as the values of bias voltage are increased, the frequency response curves bend more
Table 1. The values of design variables.

\begin{tabular}{cc}
\hline Design variable & Value \\
\hline$L$ & $100 \mu \mathrm{m}$ \\
$b$ & $30 \mu \mathrm{m}$ \\
$h$ & $1 \mu \mathrm{m}$ \\
$g_{0}$ & $1 \mu \mathrm{m}$ \\
$E$ & $1.44 \mathrm{GPa}$ \\
$\rho$ & $1000 \mathrm{~kg} / \mathrm{m}^{3}$ \\
$\varepsilon$ & $8.85 \mathrm{PF} / \mathrm{m}^{2}$ \\
$v$ & 0.38 \\
\hline
\end{tabular}

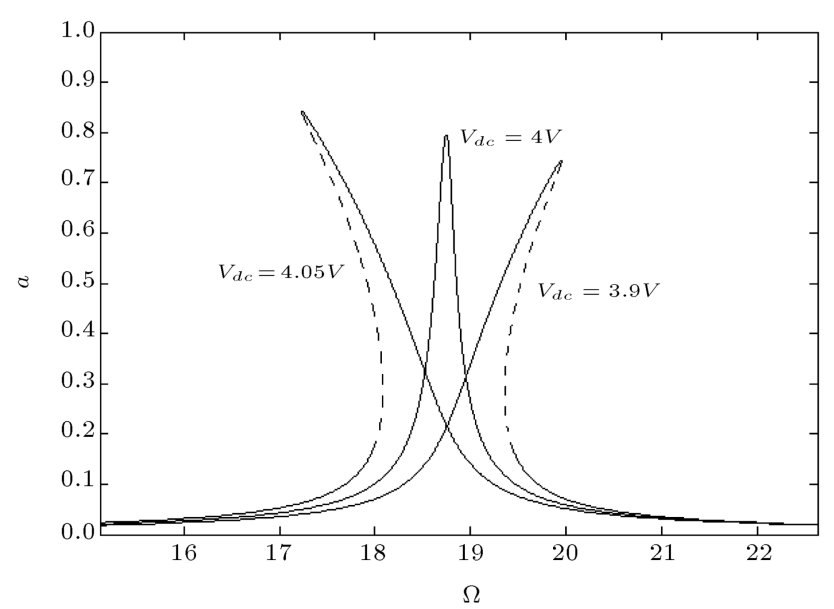

Figure 4. Frequency response of the micro-beam for various values of the $\mathrm{DC}$ voltages.

to the left, implying that the softening behavior of the system increases. Moreover, Figure 4 reveals that the softening effect of the DC voltage acts against the hardening effect of the stretching term. For voltages higher than $4 V$, the softening effect of the bias voltage overcomes the hardening effect of the stretching term, whereas, for lower values, the stretching effect can be dominated. It is shown that for a specific value of bias voltage, $V_{d c}=4 V$, the softening effect of the electrostatic loading counteracts the hardening effect of the stretching term. Consequently, the frequency response emerges without any bending and limit point bifurcation [24], "similar to a linear system".

For additional clarity, the hardening effect of the stretching term on the frequency response is shown in Figure 5, where the bias voltage is considered to be $3.5 \mathrm{~V}$. It is inferred that this effect can change the response, qualitatively, and change the bending of the frequency curves from left to right.

Taking into account the nonlocal theory, variations of the transverse vibration amplitude of the micro-beam for different nonlocal parameters, " $\mu$ ", are shown in Figure 6. In this figure, in addition to the stretching effects, the bias voltage is set as $3.5 \mathrm{~V}$. It should be noted that although the length scale 


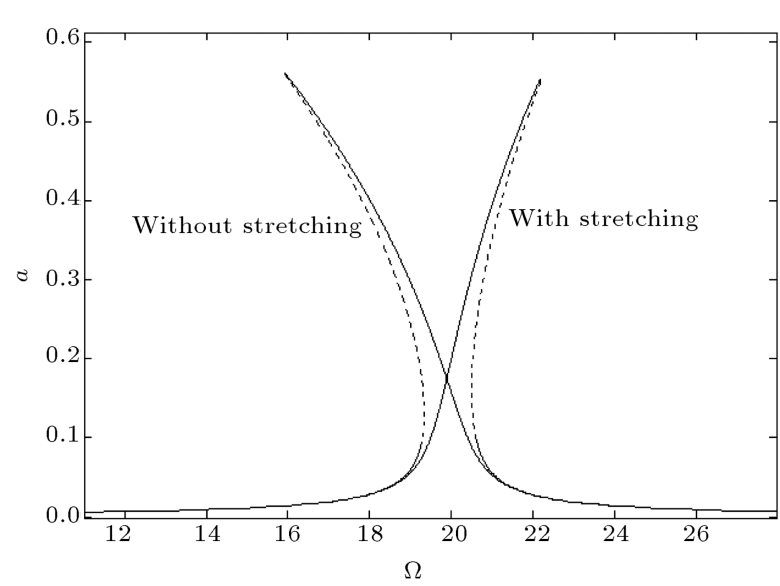

Figure 5. Comparison between the frequency response curves of the micro-beam obtained with and without the stretching effect.

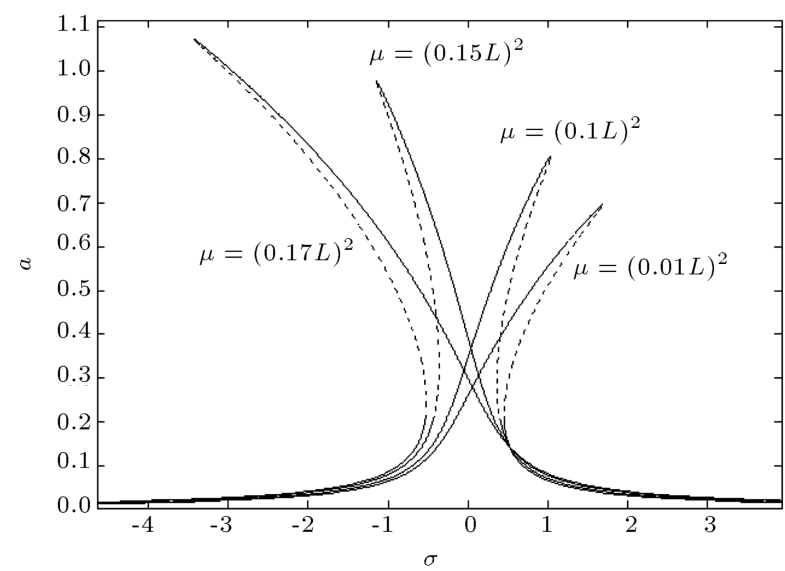

Figure 6. Frequency response curves of the micro-beam for different values of the nonlocal parameter.

parameter is fixed $(100 \mu \mathrm{m})$, the values of $\mu$ can be changed by varying the material constant " $e_{0}$ " of the micro-beam. Figure 6 shows that as the value of $\mu$ increases, the frequency response curves bend more to the left, implying that the softening behavior of the system increases. In addition, it is observed that the maximum amplitude of oscillations increase as a result of increasing the value of $\mu$.

Comparison of Figures 5 and 6 shows that the softening effect of the nonlocality is in conflict with the hardening effect of the stretching. Therefore, taking into account the stretching effects and employing the nonlocal theory for some values of the material constant, $\mu=(0.1 L)^{2}$, one can obtain a frequency curve without any bending and limit point bifurcation, "similar to a linear frequency response curve", as shown in Figure 7.

\section{Conclusion}

Employing the nonlocal theory of elasticity and taking into account the stretching effects, the nonlinear

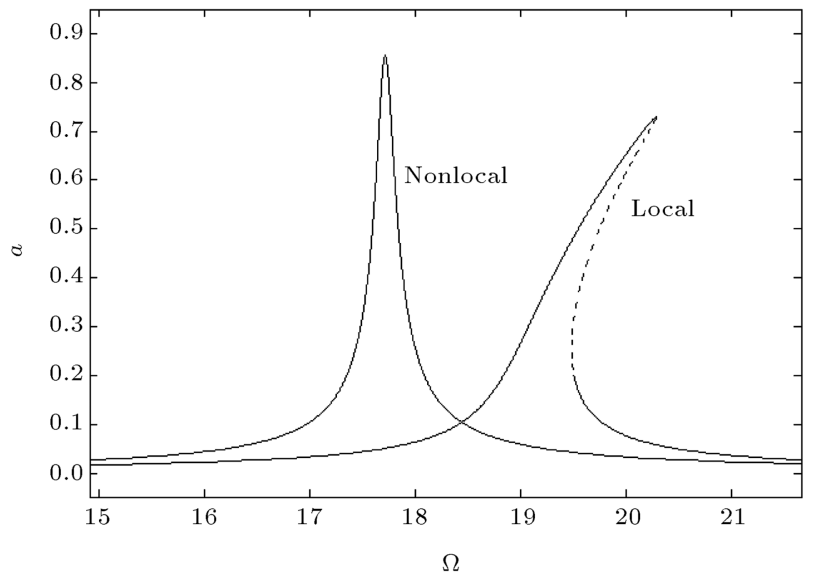

Figure 7. Effects of the nonlocality and stretching term of the frequency response of the micro-beam.

frequency response of an electrostatically actuated, double-clamped micro-beam was investigated. Taking advantage of the Galerkin projection method, the partial differential equation was discretized into a set of nonlinear, ordinary differential equations. The method of multiple scales was used to solve the equations and construct frequency response curves. The numerical results showed that the stretching term displayed hardening behavior. Examining the effects of the bias voltage on the nonlinear dynamics of the system showed that the frequency response became softer as a result of increasing the bias voltage. It was also shown that the softening behavior of the system and the maximum amplitude of the micro-beam oscillations increased with increases to the nonlocal parameter, $\mu$. The results showed that the softening effect of the bias voltage and nonlocality were in conflict with the hardening effect of the stretching. Therefore, taking into account the mentioned nonlinear effects, one can obtain a frequency curve without any bending and limit point bifurcation, "similar to a linear frequency response curve". The obtained results can be used in the design of micro-resonators in which the frequency response curves play a significant role in performance.

\section{References}

1. Lin, R. and Wang, W. "Structural dynamics of microsystems-current state of research and future directions", Mechanical Systems and Signal Processing, 20(5), pp. 1015-1043 (2006).

2. Batra, R., Porfiri, M. and Spinello, D. "Review of modeling electrostatically actuated microelectromechanical systems", Smart Mater. Struct, 16(6), pp. 2331 (2007).

3. Mobki, H., Rezazadeh, G., Sadeghi, M., VakiliTahami, F. and Seyyed-Fakhrabadi, M. "A comprehensive study of stability in an electro-statically actuated 
micro-beam", International Journal of Non-Linear Mechanics, 48, pp. 78-85 (2013).

4. Mojahedi, M., Moghimi Zand, M. and Ahmadian, M.T. "Static pull-in analysis of electrostatically actuated microbeams using homotopy perturbation method", Applied Mathematical Modelling, 34, pp. 1032-1041 (2010).

5. Seyyed Fakhrabadi, M.M., Rastgoo, A. and Ahmadian, M.T. "Analysis of pull-in instability of electrostatically actuated carbon nanotubes using the homotopy perturbation method", Journal of Mechanics of Materials and Structures, 8, pp. 385-401 (2013).

6. Nayfeh, A.H., Younis, M.I. and Eihab, M.A. "Dynamic pull-in phenomenon in MEMS resonators", Nonlinear Dynamics, 48(1-2), pp. 153-163 (2007).

7. Younis, M.I. and Nayfeh, A.H. "A study of the nonlinear response of a resonant microbeam to an electric actuation", Nonlinear Dynamics, 31, pp. 91117 (2003).

8. Nayfeh, A.H. and Younis, M.I. "Dynamics of MEMS resonators under superharmonic and subharmonic excitations", Journal of Micromechanics and Microengineering, 15(10), pp. 1840-1847 (2005).

9. Jin, Z.H. and Wang, Y.L. "Electrostatic resonator with second superharmonic resonance", Sensors and Actuators A: Physical, 64, pp. 273-279 (1998).

10. Mestrom, R.M.C., Fey, R.H.B., van Beek, J.T.M., Phan, K.L. and Nijmeijer, H. "Modelling the dynamics of a MEMS resonator: Simulations and experiments", Sensors and Actuators A: Physical, 142, pp. 306-315 (2008).

11. Rhoads, J.F., Shaw, S.W. and Turner, K.L. "The nonlinear response of resonant micro beam systems with purely-parametric electrostatic actuation", Journal of Micromechanics and Microengineering, 16, pp. 890899 (2006).

12. Chasiotis, I. and Knauss, W.G. "The mechanical strength of polysilicon films: Part 2. Size effects associated with elliptical and circular perforations", Journal of the Mechanics and Physics of Solids, 51, pp. 1551-1572 (2003).

13. Fleck, N.A., Muller, G.M., Ashby, M.F. and Hutchinson, J.W. "Strain gradient plasticity: Theory and experiment", Acta Metallurgica et Materialia, 42, pp. 475-487 (1994).

14. Kong, S., Zhou, S., Nie, Z. and Wang, K. "The sizedependent natural frequency of Bernoulli-Euler microbeams", International Journal of Engineering Science, 46, pp. 427-437(2008).

15. Akgöz, B. and Civalek, Ö. "Strain gradient elasticity and modified couple stress models for buckling analysis of axially loaded micro-scaled beams", International Journal of Engineering Science, 49, pp. 1268-1280 (2011).

16. Akgöz, B. and Civalek, Ö. "Free vibration analysis of axially functionally graded tapered Bernoulli-Euler mi- crobeams based on the modified couple stress theory", Composite Structures, 98, pp. 314-322 (2013).

17. Asghari, M., Ahmadian, M.T., Kahrobaiyan, M.H. and Rahaeifard, M. "On the size-dependent behavior of functionally graded micro-beams", Materials and Design, 31, pp. 2324-2329 (2010).

18. Ghayesh, M.H., Amabili, M. and Farokhi, H. "Nonlinear forced vibrations of a microbeam based on the strain gradient elasticity theory", International Journal of Engineering Science, 63, pp. 52-60 (2013).

19. Ghayesh, M.H., Farokhi, H. and Amabili, M. "Nonlinear dynamics of a micro scale beam based on the modified couple stress theory", Composites Part B: Engineering, 50, pp. 318-324 (2013).

20. Ma, H.M., Gao, X.L. and Reddy, J.N. "A microstructure-dependent Timoshenko beam model based on a modified couple stress theory", Journal of the Mechanics and Physics of Solids, 56, pp. 3379-3391 (2008).

21. Wang, B., Zhao, J. and Zhou, S. "A micro scale Timoshenko beam model based on strain gradient elasticity theory", European Journal of Mechanics A/Solids, 29, pp. 591-599 (2010).

22. Ansari, R., Faghih Shojaei, M., Gholami, R., Mohammadi, V. and Darabi, M.A. "Thermal postbuckling behavior of size-dependent functionally graded Timoshenko microbeams", International Journal of NonLinear Mechanics, 50, pp. 127-135 (2013).

23. Ansari, R., Gholami, R. and Sahmani, S. "Free vibration analysis of size-dependent functionally graded microbeams based on the strain gradient Timoshenko beam theory", Composite Structures, 94, pp. 221-228 (2011).

24. Civalek, Ö. and Demir, C. "Bending analysis of microtubules using nonlocal Euler-Bernoulli beam theory", Applied Mathematical Modelling, 35(5), pp. 2053-2067 (2011).

25. Civalek, Ö., Demir, C. and Akgöz, B. "Free vibration and bending analyses of cantilever microtubules based on nonlocal continuum model", Mathematical and Computational Applications, 15(2), pp. 289-298 (2010).

26. Ghayesh, M.H., Amabili, M. and Farokhi, H. "Threedimensional nonlinear size-dependent behavior of Timoshenko microbeams", International Journal of Engineering Science, 71, pp. 1-14 (2013).

27. Asghari, M., Kahrobaiyan, M.H. and Ahmadian, M.T. "A nonlinear Timoshenko beam formulation based on the modified couple stress theory", International Journal of Engineering Science, 48, pp. 1749-1761 (2010).

28. Ramezani, S. "A micro scale geometrically non-linear Timoshenko beam model based on strain gradient elasticity theory", International Journal of Non-Linear Mechanics, 47, pp. 863-873 (2012).

29. Reddy, J.N. "Nonlocal theories for bending, buckling 
and vibration of beams", International Journal of Engineering Science, 45, pp. 288-307 (2007).

30. Reddy, J.N. and Pang, S.D. "Nonlocal continuum theories of beams for the analysis of carbon nanotubes", Journal of Applied Physics, 103, p. 023511 (2008).

31. Tilmans, H.A. and Legtenberg, R. "Electrostatically driven vacuum-encapsulated polysilicon resonators. Part II. Theory and performance", Sensors and Actuators A, 45, pp. 67-84 (1994).

\section{Biographies}

Shiva Valilou received her BS degree in Mechanical Engineering from Urmia University and her MS degree from Islamic Azad University, Karaj, Iran. She is currently a PhD degree student in Mechanical Engineering at Urmia University, Urmia, Iran. Her research interests include the dynamics and stability of micro and nano structures.
Rasoul Shabani received his BS degree in Mechanical Engineering from KNT University of Technology, Iran, in 1993, and his MS and PhD degrees in 1996 and 2006, respectively, from Sharif University of Technology, Tehran, Iran. He is currently Associate Professor in the Mechanical Engineering Department at Urmia University, Iran. His research interests include fluid structure interaction, chaotic vibrations and nonlinear dynamics of MEMS/NEMS.

Ghader Rezazadeh received his BS and MS (1991) degrees in Mechanical Engineering from Isfahan Technical University, Iran, and his $\mathrm{PhD}$ degree in Applied Mechanics from Bauman Moscow State Technical University, in 1997. He is currently Professor in the Mechanical Engineering Department of Urmia University, Urmia, Iran. His research interests include stability of structures, Micro and Nano Electromechanical Systems, and Nonlinear Dynamics. 